\title{
Adapting of a Method for Qualitative and Quantitative Determination of Squalene in Distillation Cuts of Sunflower Oil
}

\author{
Irina Dubrovskaya ${ }^{1}$, Evgeniy Gerasimenko ${ }^{1}$, Margarita Slobodyanik ${ }^{1}$, and Sergey Sonin ${ }^{1}$ \\ ${ }^{1}$ Kuban State Technological University 2, Moskovskayaul., Krasnodar, 350072, Russia
}

\begin{abstract}
Squalene is a naturally-occurring dihydro-triterpene hydrocarbon $\left(\mathrm{C}_{30} \mathrm{H}_{50}\right)$ with six double bonds, which is an intermediate in the biosynthesis of phytosterol or cholesterol in plants or animals. The sources of squalene and the main methods forsqualene production and determination are consideredin brief. Sunflower oil distillation cuts have been selected as the subject of the study, since they area promising secondary raw material for the industrial squalene production. The methods of sample preparation and quantification of squalene in sunflower oil distillation cuts applying gas chromatography in combination with mass spectrometry have been adapted.

The aim of the studyis to create an integrated approach to determining the qualitative and quantitative content of squalene in distillation cuts of vegetable oils. To achieve the goal of the study, the following tasks have been solved:

- Amethodof sample preparation of distillation cuts for determination of squalene has been adapted;

- A method of qualitative and quantitative determination of squalene in distillation cuts has been modified.

As a result of this study, a technique for sample preparation of distillation cuts was proposed as well as a method for the qualitative and quantitative (absolute calibrationmethod) determination of squalene in distillation cuts of sunflower oil. To implement the technique, a Kristall 5000 gas chromatograph equipped with a mass spectrometric detector was used. Squalene and background components were recorded using the NIST 11 mass spectral database.
\end{abstract}

\section{Introduction}

Squalene is an acyclic polyunsaturated hydrocarbon triterpene $\left(\mathrm{C}_{30} \mathrm{H}_{50}-2,6,10,15,19,23\right.$-hexamethyltetracose2,6,10,14,18,22-hexaene), which contains 12 double bonds (Fig. 1). In its native form, squalene is a colourless, almost tasteless, odourless transparent liquid [1].

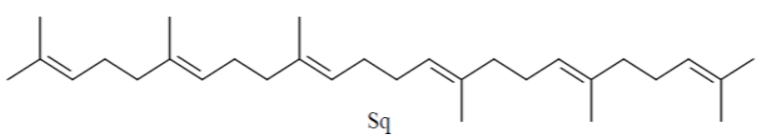

Fig. 1. Squalene structure

Squalene is a naturally-occurring bioactive compound. It plays an important role in the cholesterol biosynthesis chain, has immunomodulating, proliferative and antioxidant properties [2].

Until recently, squalene was extracted from the liver of deep-sea sharks so it was a rather expensive product. The main restrictions on the use of shark liver for squalene production are associated with environmental aspects as well as with the presence of organic pollutants (OPs) such as PCBs (polychlorinated diphenyl), PBDE (polybrominated diphenyl ether), organochlorine pesticides, polycyclic aromatic hydrocarbons, dioxin and heavy metals in seawater and their accumulation in this type of raw material [1].
All the above mentioned determined the predominance of vegetable squalene in the consumer market in most European countries as well as the expediency of searching for new promising raw materials for its production, mainly among vegetable raw materials and its secondary products.

Squalene is found in various concentrations in many types of oilseeds rawmaterials and vegetable oils extracted from them. For example, in olive, cottonseed and linseed oils, the squalene content does not exceed $0.5 \%$, while in wheat germ oil, grape seed oil, rice bran and amaranth oil, the squalene content is quite high and ranges from 2.4 to $8 \%$ [3].

Taking into account the importance of maximizing the use of raw material resources as well as the fact that during the processing of vegetable oils, squalene is removed in technological distillation processes in the composition of other relatively low-molecular compounds, distillation refining cuts are a promising secondary raw material for squalene industrial production.

Distillation cuts of vegetable oils are a complex mixture containing various valuable components such as phytosterols, tocopherols, fatty acids, sterol esters, glycerides. The content of these components varies in a wide range: tocopherols -from 1 to $20 \%$, fatty acids -from 30 to $60 \%$, phytosterols -from 5 to $30 \%$, hydrocarbons (mainly squalene)- from 10 to $30 \%$, glycerides -from 10 to $20 \%[4,5]$. 
Squalene obtaining and its use as a physiologically functional ingredient suggests the need to control its content applying effective available instrumental methods of analysis.

One of the main modern methods of analysis used for the quantitative determination of squalene is high performance liquid chromatography (HPLC), including in combination with mass spectrometry (HPLC / MS).

One of the examples of applying a technique that allows determining squalene in the presence of triglycerides is given in [6] where the use of a reverse phase HPLC method with a refractometric detector and an acetone-acetonitrile (1:1) mobile phaseare described. Another method based on size exclusion chromatography with a refractometric detector was proposed in[7]for the simultaneous determination of mono-, di-, triglycerides and squalene. The studied samples of oil are preliminarily dissolved in tetrahydrofuran and analyzed on three columns of Ultrastyragel 50,100 and $500 \AA(25 \mathrm{~cm} \times 0.77$ $\mathrm{cm})$ filled with styrene-divinylbenzene copolymer and connected in series.

Several authors [8] reported on the use of UV and diode array detectors for squalene determination. They proposed a combined HPLC / UV separation and determination method ( $\lambda: 214 \mathrm{~nm}$ - squalene; $280 \mathrm{~nm}-$ tocopherol) for the simultaneous quantitative determination of squalene and tocopherol from amaranth seed oil. It was proposed to use methanol / isopropanol / acetic acid as a mobile phase in a volume ratio of 91.95 / $8.00 / 0.05$ at a flow rate of $1.2 \mathrm{ml} / \mathrm{min}$.

In afull-scale study [9] squalene and its oxidation products were identified and analyzed by LC / APCI-MS (liquid chromatography / atmospheric pressure chemical ionization mass spectrometry). The method allows determiningsqualene epoxy and squalene and its hydroperoxides by latent fingerprints.

Along with liquid chromatography methods, there are gas chromatographic methods for the determination of squalene, but they were used for such products as amaranth seeds and vegetable oils.

Despite the wide variety of existing methods for squalene determination, all of them are rather labourious and require the use of expensive instruments and reagents.

Thus, the analysis of the existing methods for the quantitative determination of squalene showed that with regard to the distillation cuts of vegetable oils, it is necessary to adapt the sample preparation method as well as modify the gas chromatography method since it is more accessible for most analytical laboratories including industrial ones.

The purpose of the study is to develop an integrated approach to the determination of the qualitative and quantitative content of squalene in the distillation cuts of vegetable oils.

To achieve the set research goal, the following tasks were solved:

- The method of sample preparation of distillation cuts for the determination of squalene was adapted;

- A scheme for the qualitative and quantitative determination of squalene in distillation cuts was developed.
Dissolution of the sample in hexane is one of the methods of sample preparation of fatty products in order to determine squalene in them [10]. However, it should be taken into account that squalene is a liquid with a boiling point of about $280^{\circ} \mathrm{C}$, and triglycerides in fatty products have a boiling point much higher and will settle on the column without being able to evaporate, which will lead to its rapid wear.

Moreover, quantitative squalene determination by means of direct analysis is challenging due to the overlap of the components at very low concentrations. Therefore, astageofadditionalprocessingisrequired[11].

Many known methods for quantitative scalene determination describe multistage and labourious sample preparation procedures, including fractionation using column chromatography, TLC, and, in some cases, derivatization [12].

Thus, the goal of the present studies is to create an analytical approach for qualitative and quantitative determination of squalene from natural sources.

\section{Materials and methods of the study}

Distillation cuts of sunflower oil were selected as the objects of the study.

The simplest, most convenient and fairly accessible method for determining the qualitative and quantitative content of the components in a mixture is the method of gas-liquid chromatography with mass detection (GLC / MS).

The studies on the adaptation of the technique were carried out using an available Kristall 5000 chromatograph manufactured in Russia and equipped with a mass spectrometric detector.

The ionization mode of the mass spectrometer is an electron impact. Detection is carried out in selected ion mode (SIM), or by total ion current (SCAN) or in the mode of simultaneous recording of SIM / SCAN.

Separation was performed on a Zebron ZB-5MS column $30 \mathrm{~m} \mathrm{~L} \times 0.25 \mathrm{~mm} \mathrm{ID} \times 0.25 \mu \mathrm{m}$ df under the following modes:

Liquid phase: $\quad 5 \% \quad$-polysilarylene 95polydimethylsiloxane;

Temperature limits: from $60 \mathrm{C}^{\circ}$ to $325 / 350 \mathrm{C}^{\circ}$;

Chromatography conditions:

Carrier-gas is helium with a constant flow of $2.0 \mathrm{ml} /$ $\min$;

Column temperatureis $250 \mathrm{C}^{\circ}$;

Vaporizertemperature is $270 \mathrm{C}^{\circ}$;

Ionsourcetemperature is $250 \mathrm{C}^{\circ}$;

Sample injection modeissplitless;

Detection was carried out in the total ion current (SCAN) mode in the $\mathrm{m} / \mathrm{z}$ range of $40-500 \mathrm{Da}$.

For the qualitative determination of squalene, identification was carried out by comparing the mass spectra obtained during the experiment with the mass spectra of the NIST 11 mass spectra database.

A pretreatment method including saponification of the distillate and subsequent extraction of unsaponifiables with n-hexane was chosen as a sample preparation method. 


\section{Results and discussion}

At the first stage of the study, the method of gas chromatograph-mass spectrometric determination of squalene in distillation cuts was adapted. The quantitative determination of squalene was carried out using the method of absolute calibration. In this case, calibration solutions with concentrations from $1 \mathrm{mg} / \mathrm{ml}$ to $5 \mathrm{mg} / \mathrm{ml}$ were prepared from a standard sample of squalene Squalene $\geq 98 \%$, liquid from Sigma-Aldrich, which covers the entire possible range of squalene content in distillation cuts.
In the specified concentration range, the calibration curve (Figure 2) had a straight-line relationship.

As a result of the construction of the calibration curve, an approximating function was obtained and the correlation coefficient was calculated;the correlation coefficient was 1.000 , which indicates an appropriate degree of correspondence of the squalene concentration to the peak areas $(\mathrm{mg} / \mathrm{ml})$.

At the second stage of research, a sample of sunflower oil distillation cuts was prepared by saponifying $5 \mathrm{~g}$ of the sample with $50 \mathrm{~cm}^{3}$ of an alcoholic solution of potassium hydroxide with a concentration of $2 \mathrm{~mol} / \mathrm{dm}^{3}$ for $1 \mathrm{~h}$.

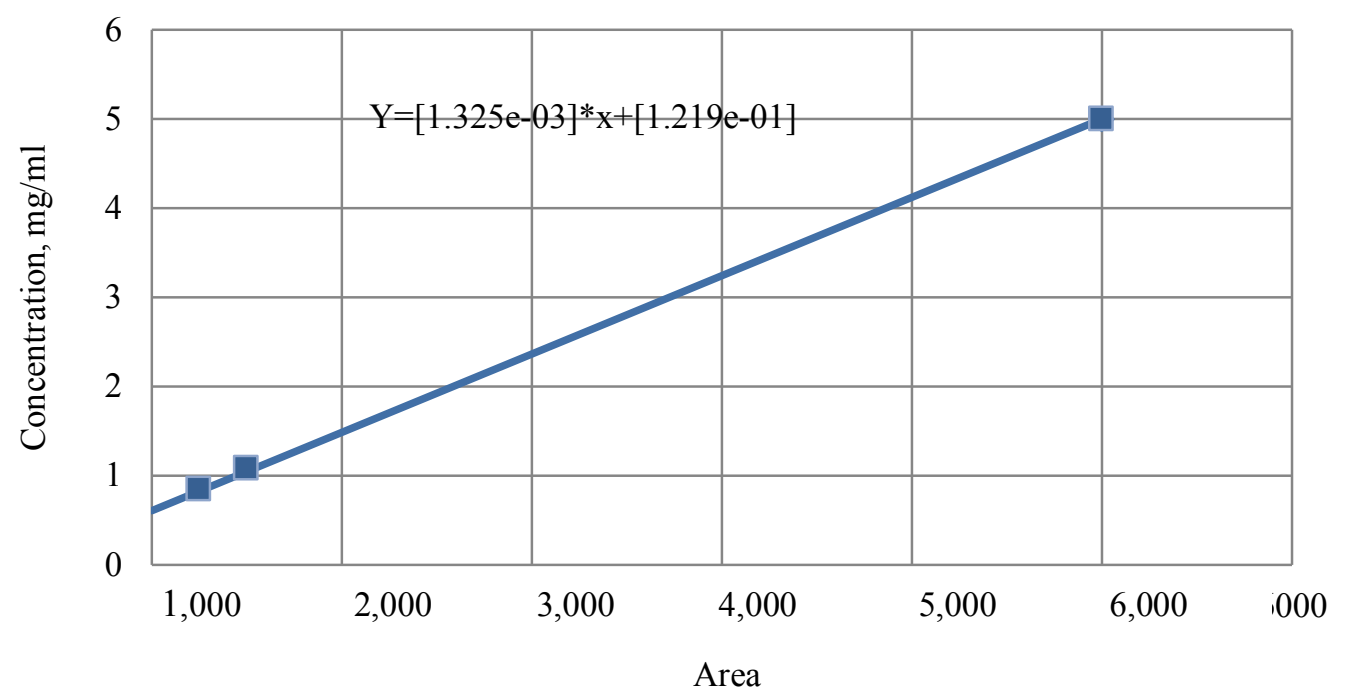

Fig. 2. Calibrationcurveofthe dependence of the peak area on the concentration of squalene

Then, $50 \mathrm{~cm}^{3}$ of water was added; the contents of the flask were cooled, quantitatively transferred to a separatory funnel, and the flask was rinsed several times with hexane (the total volume was $50 \mathrm{~cm}^{3}$ ). All rinsing portions of hexane were transferred into the same separatory funnel, shaken vigorously for $1 \mathrm{~min}$. Extraction with hexane was carried out 6 times. The combined hexane extracts were first washed with weakly alkaline 50\% alcohol, and then, to remove soap residues, they were repeatedly washed with $25 \mathrm{~cm}^{3}$ portions of $50 \%$ alcohol (without alkali) until phenolphthalein ceased to give a red colouration of the washing liquid (previously diluted with double-triple volume of water).

The washed hexane extract was quantitatively transferred into a pre-dried and weighed flask, pouring the solution through a funnel with a paper filter.

Hexane was distilled off on a rotary evaporator. The resulting residue was dried at the temperature of $80^{\circ} \mathrm{C}$ to a constant weight; weighing was carried out every $15 \mathrm{~min}$.

The resulting non-saponifiable fraction was dissolved in $50 \mathrm{~cm}^{3}$ of hexane and $5 \mu \mathrm{l}$ of the resulting solution was subjected to chromatography. The chromatogram of sunflower oil distillation cuts is given in Figure 3. 


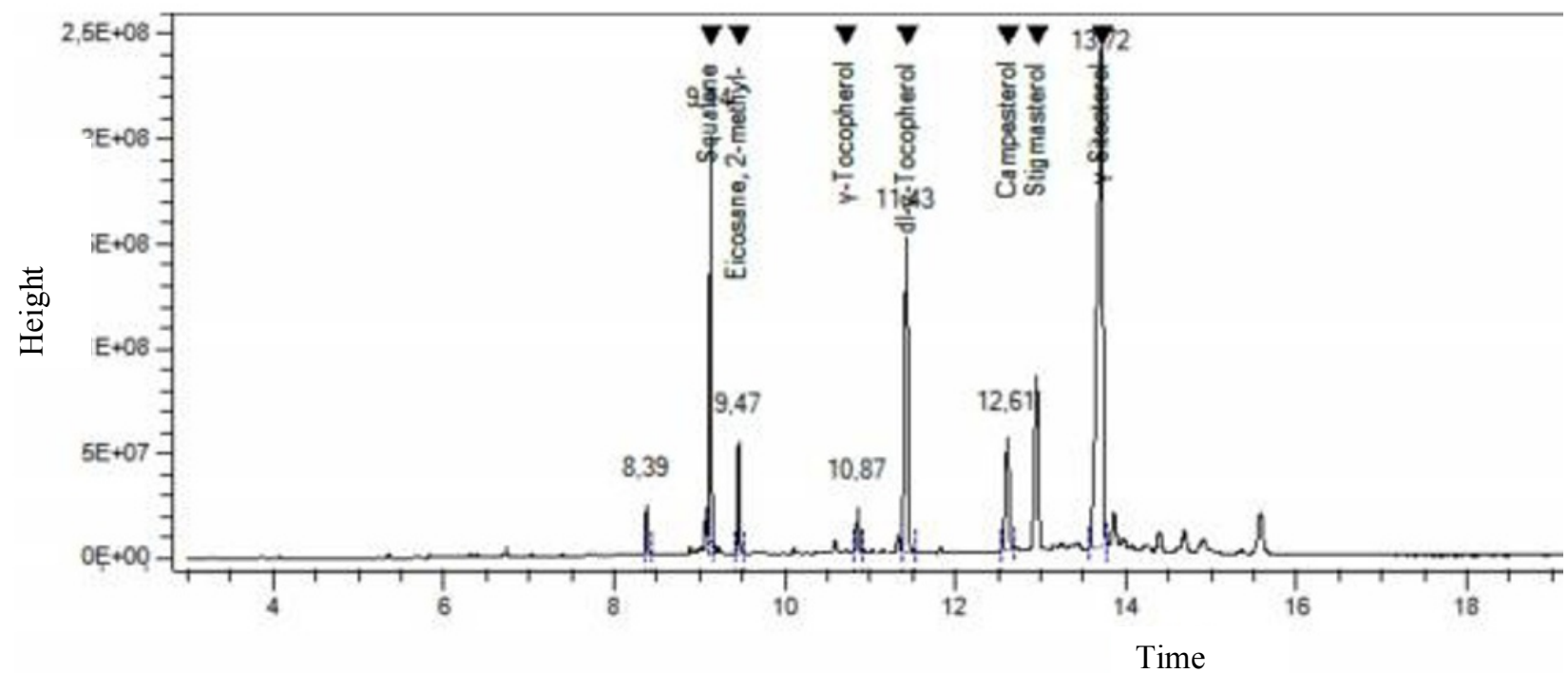

Fig. 3. Chromatogram of a sample of distillation cuts

In the specified subject of thestudy, in addition to squalene, the following dominant components were found: eicosenic acid, tocopherol, campesterol, stigmasterol, and $\beta$-sitosterol.

The results are given in the following table 1 .

Table 1. Component composition of distillation cuts

\section{Таблица кандидатов}

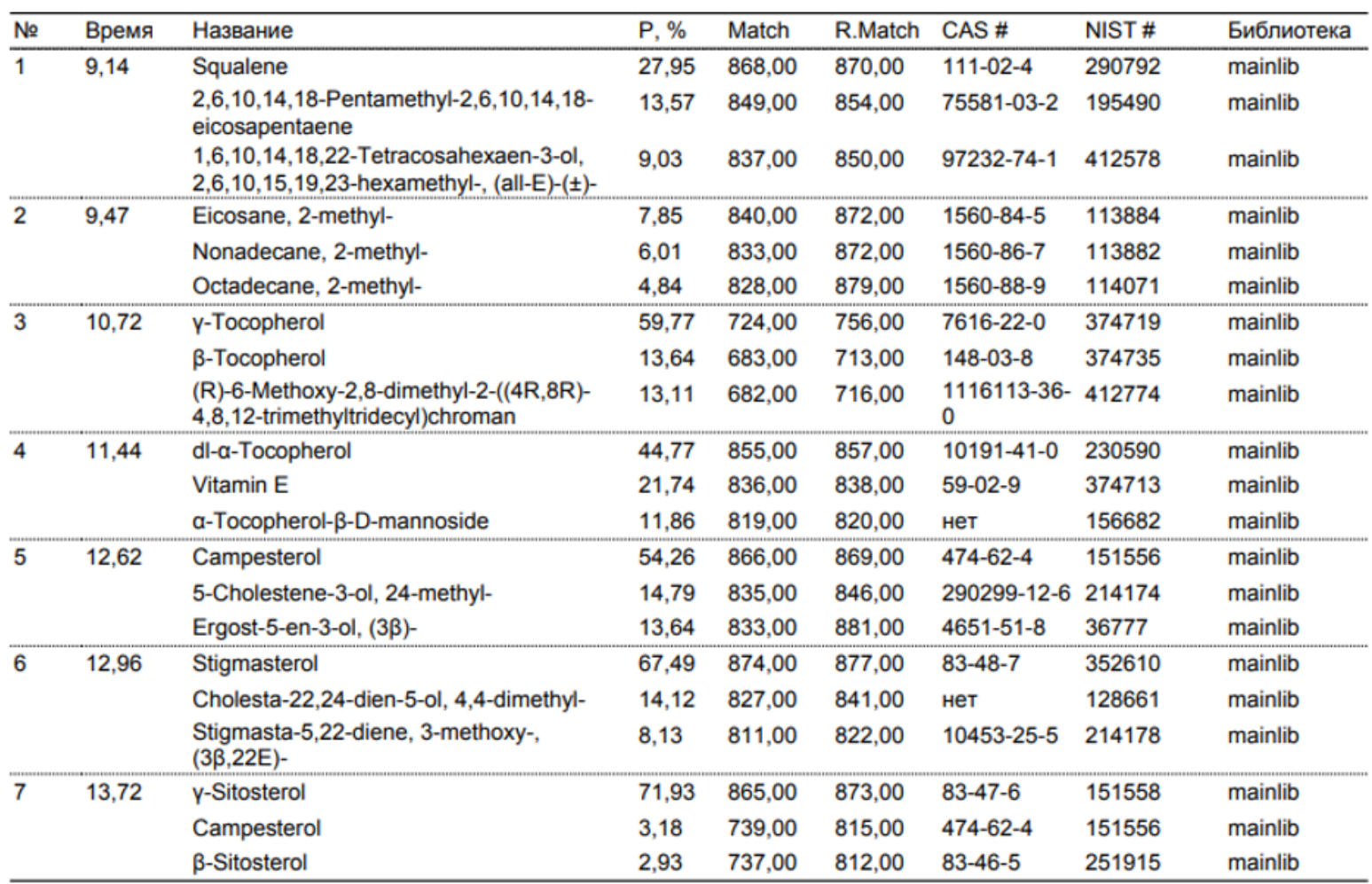

\section{Conclusion}

As a result of this study, a method for sample preparation of distillation cuts has been proposed as well as a method for qualitative and quantitative (method of absolute calibration) detection of squalene in distillation cuts of sunflower oil has been developed. The gas chromatograph-mass spectrometric method was used as an analytical tool. Squalene and background components were recorded using the NIST 11 mass spectra database.

The study was carried out within the framework of the state assignment of the Ministry of Education and Science of the Russian Federation, FZEZ-2020-0004 project. 


\section{References}

1. Shark squalene. URL: http://skvalene.ru/svoystvaakulego-skvalena.html (date of access: August 6.08. 2016).

2. I.M. Magomedov, T.V. Chirkova, A.I. Chirkova Int. $\mathrm{j}$ of appl. and fundam. res. Squalene as an antihypoxant in animals and plants 590 (2016)

3. Qualitative and quantitative determination of squalene in oils and reaction mixtures by HPLC method URL:

http://cyberleninka.ru/article/n/kachestvennoe-ikolichestvennoe-opredelenie-skvalena-v-maslah-ireaktsionnyh-smesyah-metodom-vezhh (date of access: 8.06.2016).

4. E. A. Butina, E. O. Gerasimenko, I.A. Bugaets, B.E. Krasavtsev, I.A. Dubrovskaia J. Pharm. Sci. \& Res. Features of the main components of vegetable oils' distillates 10(1) 118 (2018)

5. E.A. Butina, E.O. Gerasimenko, S.A. Kalmanovich, O.S. Vorontsova, N.V. Alpatova, J of Pharm. Sci. and Res. Features of vegetable oils' distillates obtained by Russian oil processing enterprises 10(1) 65 (2018)

6. N. Cortesi, P.A. Rovellini, E. Fedeli, Controllodellagenuinità, indaginesulla qualità Rivista Italiana Delle Sostanze Grasse La cromatografia liquida nell'analisidei lipidi 73(9) 397 (1996)

7. M.V. Ruiz-Mendes, M.K. Dobarganes, Food chem. A combination of chromatographic methods for the analysis of complex deodorant distillates from edible oil processing 103(4) 1502 (2007)

8. H. Sun, D. Wiesenborn, K. Tostenson, J. Gillespie, P. Rayas-Duarte, J of the Am. Oil Chem. Soc. Fractionation of Squalene from Amaranth SeedOil. 74:413-418 (1997).

9. K.A. Mountfort, H. Bronstein, N. Archer, S.M. Jickells, Analitical Chemistry Identification of oxidation products of squalene in solution and in latent fingerprints by ESI-MS and LC/APCI-MS 79(7) 2650 (2007)

10. D.I. Pisarev, O.O. Novikov, M.A. Bocharnikova, Yu.G. Vasilyeva, A.Yu. Malyutina, Scientific Res. Med. and pharm. Development of a method for determining the content of squalene in some vegetable fatty oils 24 (2016)

11. K.N. Desai, H. Wei, C.A. Lamartiniere, Cancer Lett The preventive and therapeutic potential of the squalenecontaining compound. Roidex on tumor promotion and regression 10193 (2014)

12. J. C. Sulpice, J. Ferezou Squalene isolation by HPLC and quantitative comparison by HPLC and GLC. Lipids 196312014 